\title{
Analysis of Genetic Diversity of Trypanosoma cruzi: an Application of Riboprinting and Gradient Gel Electrophoresis Methods
}

\author{
JR Stothard ${ }^{+}$, IA Frame, HJ Carrasco, MA Miles
}

\begin{abstract}
Pathogen Molecular Biology and Biochemistry Unit, Department of Infectious and Tropical Diseases, London School of Hygiene and Tropical Medicine, Keppel Street, London WC1E 7HT, UK
\end{abstract}

\begin{abstract}
Analysis of restriction fragment length polymorphism (RFLP) profiles derived from digestion of polymerase chain reaction (PCR) products of the ribosomal $18 S$ from Trypanosoma cruzi yields a typical 'riboprint' profile that can vary intraspecifically. A selection of 21 stocks of $\mathrm{T}$. cruzi and three outgroup taxa: T. rangeli, T. conorhini and Leishmania braziliensis were analysed by riboprinting to assess divergence within and between taxa. T. rangeli, T. conorhini and L. braziliensis could be easily differentiated from each other and from $\mathrm{T}$. cruzi. Phenetic analysis of PCR-RFLP profiles indicated that, with one or two exceptions, stocks of $T$. cruzi could be broadly partitioned into two groups that formally corresponded to T. cruzi I and T. cruzi II respectively. To test if ribosomal $18 S$ sequences were homogeneous within each taxon, gradient gel electrophoresis methods were employed utilising either chemical or temperature gradients. Upon interpretation of the melting profiles of riboprints and a section of the 18S independently amplified by PCR, there would appear to be at least two divergent $18 S$ types present within T. cruzi. Heterogeneity within copies of the ribosomal $18 S$ within a single genome has therefore been demonstrated and interestingly, this dimorphic arrangement was also present in the outgroup taxa. Presumably the ancestral duplicative event that led to the divergent $18 S$ types preceded that of speciation within this group. These divergent 185 paralogues may have, or had, different functional pressures or rates of molecular evolution. Whether or not these divergent types are equally transcriptionally active throughout the life cycle, remain to be assessed.
\end{abstract}

Key words: riboprinting - 18S - paralogy - denaturing gradient gel electrophoresis temperature gradient gel electrophoresis

At the molecular DNA level, the ability to characterise stocks, strains and clones of kinetoplastid protozoa is fundamental to understanding the epidemiology of their respective diseases (Tibayrenc 1998a) and that of Trypanosoma cruzi and Chagas disease presents no exception (Macedo \& Pena 1998). For most simplistically, molecular markers discriminate and define clones or strains of the pathogen, providing taxonomic characters, upon which further DNA assays can be developed e.g. sensitive tools for diagnostics. By the shared presence of taxonomic characters, molecular data have the potential to infer evolu-

Funded by The Wellcome Trust.

${ }^{+}$Corresponding author. Fax: +442079425518. E-mail: r.stothard@nhm.ac.uk

Present address: Biomedical Parasitology Division, Department of Zoology, The Natural History Museum, Cromwell Road, London SW7 5BD, UK

Received 13 April 2000

Accepted 15 May 2000 tionary relationships or, at the very least, groups of organisms on the basis of similarity i.e. their phenetic patterns ( $\mathrm{Li}$ 1997). Over the last 10 years, DNA typing of kinetoplastid parasites has undergone impressive developments, primarily accruable to development and implementation of polymerase chain reaction (PCR) based assays. So much so that a new synthetic field of research has been defined and generically known as 'integrated genetic epidemiology of infectious diseases' (Tibayrenc 1998b).

As researchers attempt to find natural 'demes' or divisions, the taxonomy within and between the isolates that are collectively known as $T$. cruzi is attracting considerable attention. Substantial heterogeneity is known to exist but there has been a general reluctance to name formal taxa (Momen 1999). The partial realisation of the divisions, however, has led to the nomenclature of $T$. cruzi I, $T$. cruzi II and $T$. cruzi to try to acknowledge formally this intraspecific variation (Anon 1999). T. cruzi I is equivalent to principal zymodeme $1(\mathrm{Z1})$ and $T$. cruzi II is equivalent to principal zymodeme 2 (Z2) defined by Miles et al. (1977). Those strains that 
exhibit hybrid-like characters, or await further characterisation, or are from principal zymodeme 3 (Z3) (Miles et al. 1981) are collectively referred to as $T$. cruzi [without the group designation suffix (Anon 1999)]. Z2 isolates were originally described from central and eastern Brazil, in domestic transmission cycles, whereas $\mathrm{Z1}$ isolates were predominantly sylvatic when found sympatric with $\mathrm{Z} 2$ but in the north of the Amazonian basin were in both domestic and sylvatic cycles. Z3 isolates were rarely isolated from humans and are almost exclusively associated with burrowing animals such as the armadillo. In conjunction with the problematic strain taxonomy, the reproductive biology of $T$. cruzi is also complex. Clonal propagation of T. cruzi is thought to predominate (Tibayrenc 1998a) though there is evidence of sexuality in sylvatic transmission cycles (Bogliolo et al. 1996, Carrasco et al. 1996) and evidence of genetic exchange has been found in the laboratory (Stothard et al. 1999).

This paper assesses the impact that molecular characterisation of the $18 \mathrm{~S}$ gene has had upon strain typing of $T$. cruzi. The paper draws upon recent work and focuses upon methods of gradient gel electrophoresis, which are also known as mutation analysis.

\section{RIBOPRINTING}

Analysis of sequence variation within the ribosomal $18 \mathrm{~S}$ has proven useful for phylogenetic purposes within the Trypanosomatidae (Noyes 1998, Briones et al. 1999, Stevens \& Gibson 1999, Wright et al. 1999), though the use of this region for phylogenetic inference has not been without debate (Stothard in press). In T. cruzi, there are approximately 110 copies of the $18 \mathrm{~S}$ per nucleus, the organisation of which is slightly unusual in comparison to other protozoa (Pulido et al. 1996). On a less finer scale than DNA sequencing, variation within the $18 \mathrm{~S}$ can be detected by restriction enzymes since many enzymes, especially those with four base pair recognition sites e.g. AluI, have several cutting sites within the $18 \mathrm{~S}$. By using a battery of such frequently cutting enzymes, the $18 \mathrm{~S}$ can be easily 'typed' and quickly scanned for variable nucleotides without the need for DNA sequencing. Moreover, through the use of polymerase chain reaction (PCR), the amplification products can be directly digested and separated by conventional agarose or polyacrylamide electrophoresis to produce a 'fingerprint-like' profile that can be easily, cheaply achieved within a single working day. Indeed as riboprinting is less sensitive than DNA sequencing, this might even be an advantageous way to analyse variation within the $18 \mathrm{~S}$. For example, as the $18 \mathrm{~S}$ has been shown to be paralogous (Stothard et al. 1997); as long as the enzyme cutting sites do not fall within these paralogous regions, the associated problems of paralogy/orthology disappear (Stothard in press). In contrast, if the restriction sites were in the paralogous regions, the riboprint profile could be interpreted as an incomplete digestion (Fig. 1).

The process of generating PCR-RFLP (restriction fragment length polymorphism) profiles from the $18 \mathrm{~S}$ is also known as riboprinting (Clark 1992). Riboprinting allows detection of cryptic genetic variation within species, organism misidentifications and culture mix-ups, independent verification of DNA sequences, and the rapid generation of data useful in phylogenetic analyses (Clark et al. 1995). Pioneering studies using this methodology revealed variation between species of Entomoeba (Clark \& Diamond 1991) and was later applied upon sylvatic strains of $T$. cruzi from North America, coining the nomenclature of 'ribodemes' to group isolates of $T$. cruzi with the same riboprint (Clark \& Pung 1994). Stothard et al. (1998a) expanded the number of isolates examined by riboprinting to 21 , with better coverage of $\mathrm{Z3}$. Through the inclusion of outgroup taxa T. rangeli, T. conorhini and Leishmania braziliensis an assessment was made of the evolutionary divergence within T. cruzi and to explore the ability of riboprinting to be used as a diagnostic assay. For example, $T$. rangeli is transmitted by the same triatomine vectors as $T$. cruzi and mixed infections may occur in both vertebrate and invertebrate hosts, however, only $T$. cruzi infections are pathogenic in man (Hoare 1972). The $18 \mathrm{~S}$ was amplified, using the primers SSU1-5' GATCTGGTTGATT CTGAA and SSU2-5' GATCCAGCTGCAGGTT CA, to produce a fragment of approximately 2000 bp. A selection of 10 restriction enzymes was used to sequentially digest the $18 \mathrm{~S}$ and RFLP patterns were revealed by PAGE and silver staining (Stothard et al. 1998a). Both interspecific and intraspecific variation were revealed. For example, digestion with $C f o$ I differentiated $T$. cruzi from the other species: T. rangeli, $T$. conorhini and $L$. braziliensis (Stothard et al. 1998a). To date, CfoI riboprint profiles within $T$. cruzi were invariant and would suggest that this profile is 'stable' for all $T$. cruzi isolates, though examination of further isolates is warranted. In contrast, digestion with HaeIII detected variation within $T$. cruzi that differentiated Z1 from Z2/Z3 on the presence and absence of a fragment of approximately $840 \mathrm{bp}$ respectively. As such, the $18 \mathrm{~S}$ shows promise as a good target for diagnostic PCR methods as parasite detection could be simultaneous with stock typing (Stothard et al. 1998a). 
After pooling the riboprint profiles generated from the 21 stocks of $T$. cruzi, an estimate of pairwise phenetic distance between stocks of $T$. cruzi was calculated (Stothard et al. 1998a). This data matrix was analysed by principal coordinate analysis and relationships between isolates were plotted for the first three factors that summarised $85 \%$ of the total variance. Interestingly a bi-polar division within $T$. cruzi was detected, with partitions broadly corresponding to $\mathrm{Z} 1$ ( $T$. cruzi I) and $\mathrm{Z} 2$ ( $T$. cruzi II). The position of certain isolates, however, was less clear-cut. For example, for Z3 isolates, whilst CANII and CANIII were placed nearer to $\mathrm{Z} 2$ isolates than $\mathrm{Z} 1$, both BugCN33 and BugCN35 remained almost equidistant between $\mathrm{Z} 1$ and Z2. The affiliations of these two isolates within
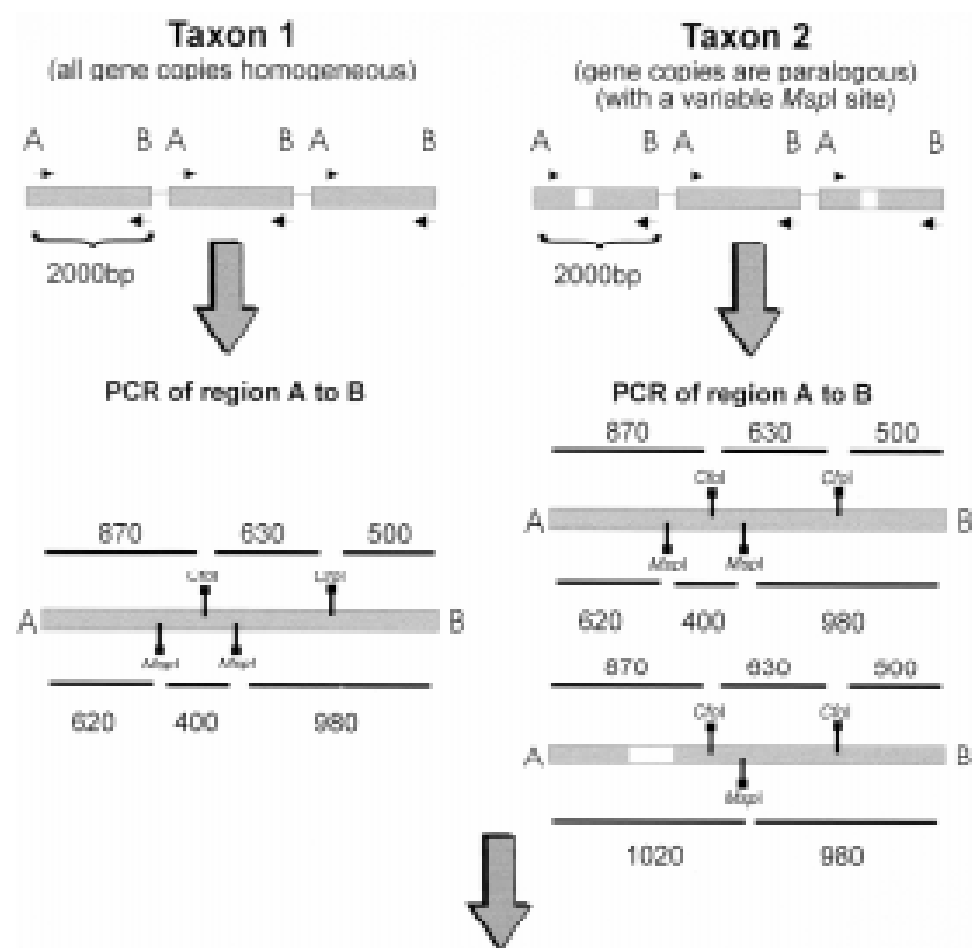

\section{Schematic gel of RFLP profiles}

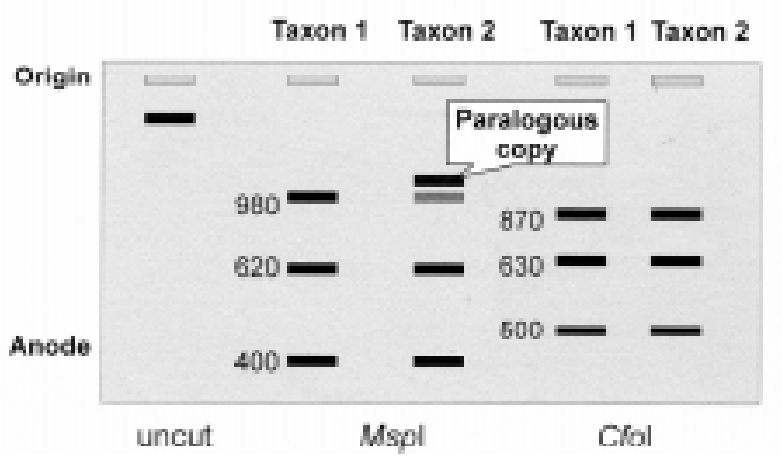

Fig. 1: schematic representation of the problem of gene paralogy within a hypothetical gene family using restriction fragment length polymorphism (RFLP) studies. Two taxa, taxon 1 and taxon 2, are presented. The tandemly repeated genes within the gene family of taxon 1 are homogeneous (uniformly grey) such that the RFLP patterns for MspI and $C f o I$ are the same for the repetitive copies. In taxon 2, however, the gene array is not homogeneous. The white region (of approximately $200 \mathrm{bp}$ ) is divergent between the repetitive copies. Whilst the $C f o$ I profile is constant between the copies, as there are no restriction sites within this divergent region, the MspI profile is variable. The white region has lost a MspI cutting site and in addition, is considerable different to the comparable region of the DNA sequence of grey. The MspI RFLP profile of taxon 2 might be interpreted as an incomplete digestion but in reality is direct evidence for gene paralogy. RFLP data generated from cutting sites outside the white region would be free from evolutionary paralogy but those within are not. Minor bands in riboprints might be good evidence for such paralogous variation [e.g. see Fig. 1 A of Clark \& Pung (1994)]. 
this bi-polar grouping remained ambiguous and perhaps if there was better taxonomic sampling of such isolates they may even form a new, coherent group. Similarly the relationship of CL Brener is somewhat speculative as it remained singularly placed (Stothard et al. 1998a).

\section{GRADIENT GEL ELECTROPHORESIS}

A variety of advanced electrophoretic methods can be used to screen DNA sequences that are obtained by conventional PCR, for sequence changes by direct physical separation (Cotton 1997). These methods for mutation detection are also collectively known as mutational analysis and include, amongst others, single strand conformational polymorphism (SSCP) analysis and gradient gel electrophoresis (GGE). Collectively these methods share the ability to separate DNA fragments of identical size but of differing sequence that would otherwise co-migrate during conventional electrophoretic separation. These methods, whilst widely applied in biomedical research, are beginning to be adopted within parasite strain typing (Stothard et al. 1997, Gasser \& Zhu 1999).

Typically, a double stranded DNA fragment has a characteristic melting profile upon the disassociation of this homoduplex into its respective single stranded chains (Cotton 1997). This melting profile is reversible, giving rise to a sigmoid transition curve (Fig. 2A). Double stranded DNA mobility is greater than that of single stranded or partially melted DNA. This profile is also sequence dependent, such that differing DNA sequences have different melting profiles despite being identical in length (Fig. 2B). Gradient gels can utilize either a chemical (DGGE) or temperature (TGGE) method. Gels can also be perpendicular (the gradient is at right angles to the direction of electrophoresis; Fig. 2A, B) or parallel (the gradient is in the same direction of electrophoresis; Fig. 2C). The precise gradient is usually determined empirically but computer programs, such as MELT 87 (Lerman $\&$ Silverstein 1987), can predict the behaviour of a specified sequence and an optimal gradient (Cotton 1997). Gradient gel electrophoresis can detect single point mutations between test and reference DNA. It is also a good method to separate complex mixtures of DNA that would otherwise comigrate during conventional electrophoresis. As such, gradient gel methods are ideally suited to study the variation in gene families such as the ribosomal RNA complex (Schlötterer 1995).

\section{DENATURING GRADIENT GEL ELECTROPHORESIS (DGGE)}

DGGE has the ability to separate DNA fragments of identical size but differing sequence identity through the utilization of a chemical gradient, i.e. urea and formamide. This gradient is formed upon casting the polyacrylamide gel and after gel polymerization the gradient remains stable during subsequent electrophoresis. DGGE was used to analyze a portion of the $18 \mathrm{~S}$ amplified from a small selection of stocks of T. cruzi (Stothard et al. 1997). PCR primers flanking the V1 and V2 domains of the $18 \mathrm{~S}$ were used and the 5' forward primer was GC-clamped to enhance the sensitivity of the technique (Sheffield et al. 1992). Primer sequences in the 5' to 3' orientation were as follows: SSUCLPCGCCCGCCGCCCCGCGCCCGGCCCGCC GCCCCCGCCCGGCAGGAATCTGCGCATGGCT and SSUREV-GTCAACGCCATGGCAGTCCAC.

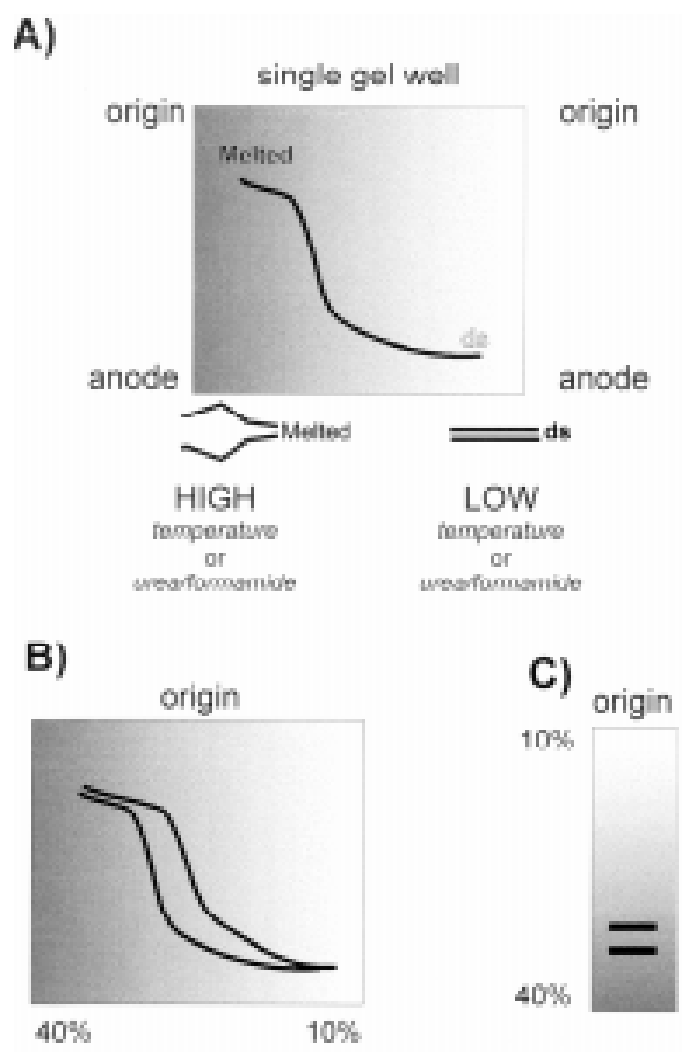

Fig. 2: basic theory of gradient gel electrophoresis. A: a chemical or temperature gradient is formed perpendicular to the direction of electrophoresis. A DNA fragment is loaded across the single well and migrates towards the anode. Where the gradient is insufficient to melt the fragment, the mobility is the same as that of double stranded (ds) DNA. As the gradient increases the fragment progressively melts and is electrophoretically retarded. The melting curve (sigmoidal in this instance) is dependent upon the sequence composition; B: two DNA fragments of identical size, but differing sequence, will have different melting profiles. This electrophoretic mobility shift allows separation of the two types; C: to facilitate further comparison (i.e. lane to lane), parallel gels (gradient in the same direction of electrophoresis) are used. The two fragments illustrated would co-migrate under standard PAGE or agarose electrophoresis, however, gradient gels permit direct separation. 
Upon non-denaturing PAGE, a single 450bp amplification product was seen and no size variation was apparent between taxa (Stothard et al. 1997). Through the use of perpendicular gels, a chemical gradient from $10 \%$ to $25 \%$ was shown to denature the fragment. The sigmoidal transition profile is illustrative of a single melting domain within the amplified fragment; in this particular instance the PCR product was derived from a bacterial plasmid carrying the $18 \mathrm{~S}$ from T. cruzi (Fig. 3A).

PCR products were then directly obtained from PCR amplification from $T$. cruzi genomic DNA preparations. Parallel gels that covered the same chemical gradient range (Fig. 3B) then separated these products. Variation was immediately detectable between the homoduplex molecules of Z1/Z3 and Z2. As two bands were apparent in each lane, there would appear to be at least two divergent $18 \mathrm{~S}$ sequence types present within each stock examined. SSCP analysis could also detect variation between Z1/Z3 and Z2. By using heteroduplex analysis (Stothard et al. 1997), a finer level of resolution was achieved by finding variation between $\mathrm{Z} 1$ and $\mathrm{Z} 3$. In addition, as extra-bands were visualized upon heteroduplex analysis of $Z 1$ and $Z 2$, the presence of at least two $18 \mathrm{~S}$ divergent types within each stock was further confirmed. More importantly each of these two types differed between stocks (Fig. 3B).

\section{TEMPERATURE GRADIENT GEL ELECTROPHORE- SIS (TGGE)}

To further screen the whole $18 \mathrm{~S}$ gene for additional regions of paralogy, riboprint profiles were subjected to TGGE analysis. Riboprint profiles, obtained from seven enzymes: HaeIII, AluI, Sau3A, ScrFI, MspI, DdeI and CfoI, were separated by TGGE covering a thermal gradient from $45^{\circ} \mathrm{C}$ to $65^{\circ} \mathrm{C}$. Riboprint profiles from $C f o \mathrm{I}$ and DdeI exhibited split melting curve profiles characteristic of regions of paralogy. Unfortunately, as the fragment shown to be paralogous for DdeI digestion was not conserved across $T$. cruzi as there was RFLP variation, this profile was not examined further (Stothard et al. 1998b). The riboprint profile for $C f o \mathrm{I}$ was invariant within $T$. cruzi and was therefore further characterized. In total, five isolates of $T$. cruzi covering the principal zymodemes and $T$. conorhini, $T$. rangeli and $L$. braziliensis were shown to exhibit a profile indicative of paralogy [see fragment B in Fig. 1, Fig. 2 (Stothard et al. 1998b)].

To ascertain the region of the $18 \mathrm{~S}$ that was shown to be paralogous, TGGE profiles were electroblotted onto nylon membranes and followed by hybridization with the $18 \mathrm{~S}$ V1, V2 regions characterized by DGGE analysis (see above). The frag- ment, labeled B, was shown to hybridize therefore the paralogous regions of the $18 \mathrm{~S}$ were presumably confined only to the V1, V2 regions. Interestingly, this gene dimorphism is present in $T$. rangeli, T. conorhini and Leishmania spp. For Leishmania, however, there would appear additional regions of paralogy within the 18S [see fragment labeled * Fig. 2 (Stothard et al. 1998b)]. This fragment clearly contains three divergent types. What is more, in consideration with linkage to the dimorphic V1, V2 regions, there could be up to six

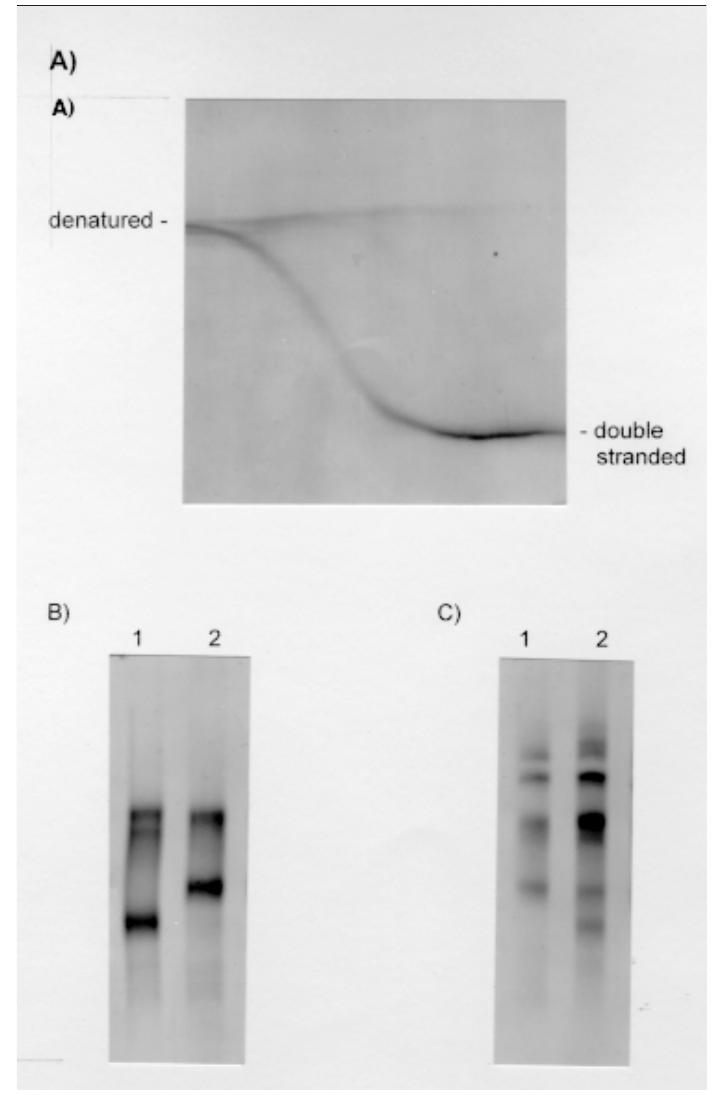

Fig. 3: denaturating gradient gel electrophoresis analysis of ribosomal polymerase chain reaction (PCR) products from Trypanosoma cruzi shows that two $18 \mathrm{~S}$ types are present; A: perpendicular analysis of the melting profile of an amplification product derived from a cloned $18 \mathrm{~S}$ in a bacterial plasmid. Increasing chemical gradient occurs from right to left across the gel. A single melting domain is inferred as the profile is sigmoidal in shape; B: parallel gradient gel analysis of PCR products from $T$. cruzi genomic preparations. Lane 1 a Z2 stock, lane 2 a Z1 stock. There is a clear mobilty difference between the two stocks and at least two, possibly three 18S types (lane 1) are present; C: heteroduplex analysis of PCR products. Heterduplexes are formed by mixing PCR products, denaturing then subsequently allowed to cool (Stothard et al. 1997). Lane 1 a Z1/Z1 stock combination, lane 2 a Z2/Z1 stock combination. The profiles are identical to that of Fig. B with the exception that heteroduplex molecules (retarded to the homoduplex molecules) are now seen. 
divergent types present within the genome of $L$. braziliensis. If we assume that Leishmania was ancestral to other trypanosomes, it would appear that at least two divergent $18 \mathrm{~S}$ types have been lost within Trypanosoma. Conversely, if we infer that if Trypanosoma was ancestral, Leishmania would require two new duplicative events to give rise to these paralogous copies.

\section{CONCLUSIONS}

The ability to differentiate T. cruzi with the ribosomal 18S has shed new light on the interpretation of the evolutionary relationships within the trypanosomes. The assumption that this gene family is homogeneous within a genome is clearly misleading and the dynamics of this gene family are more complicated than prevously thought. Firstly the problem of sequence paralogy is a fundamental issue within this group. The shared duplicative state of the V1, V2 regions would appear to be ancestral (plesiomorphic) to the group as a whole. Interpretation of the analysis of Briones et al. (1999) is particularly interesting in this light; the separate analyses of the variable regions of the $18 \mathrm{~S}$ derived conflicting tree topologies, indicating substantive rate heterogeneity across the $18 \mathrm{~S}$. This might not be unexpected given that certain regions of it seem to be afflicted by evolutionary paralogy. Secondly, the polarity of interpretation of the $18 \mathrm{~S}$ in terms of duplications or losses of the divergent types needs further clarification. Most pessimistically, how confident can we be of the current $18 \mathrm{~S}$ data seeing as T. cruzi could have lost two thirds of the molecular evidence?

The relative insensitivity of riboprinting therefore has advantages in light of this sequence paralogy. The restriction sites studied so far appear to fall outside these paralogous regions and without recourse to the gradient gel methods their very existence might have remained undetected. In explanation, the contemporary way to derive $18 \mathrm{~S}$ sequence data is to pool several PCR products and cycle sequence the template using overlapping sets of primers. The occurrence of split chromatogram peaks may well have gone unnoticed seeing as certain templates can be preferentially amplified/sequenced in comparison to the mixture as a whole. Riboprinting confirms the existence of two biological lineages within $T$. cruzi, corresponding to $T$. cruzi $\mathrm{I}$ and $T$. cruzi II, but indicates that if there were greater taxonomic sampling especially of Z3, new groups might come to light. Perhaps if riboprinting, using only HaeIII and CfoI, were to be used in conjunction with the DNA typing assays of the 24S D7 domain by Souto et al. (1999) this combined methodology could lead to a very powerful diagnostic tool for nearly all kinetoplastid infections. It would be interesting to explore the potential of these assays in a more clinical setting.

\section{ACKNOWLEDGEMENTS}

To Jamie Stevens for his invitation to the 'Workshop on Molecular Evolution of Trypanosomes'.

\section{REFERENCES}

Anon 1999. Recommendations from a Satellite Meeting. Mem Inst Oswaldo Cruz 94: 429-432.

Bogliolo AR, Lauria-Pires L, Gibson WC 1996. Polymorphisms in Trypanosoma cruzi: evidence of genetic recombination. Acta Trop 61: 31-40.

Briones MRS, Souto RP, Stolf BS, Zingales B 1999. The evolution of two Trypanosoma cruzi subgroups inferred from rRNA genes can be correlated with the interchange of American mammalian faunas in the Cenozoic and has implications to pathogenicity and host specificity. Mol Biochem Parasitol 104: 219-232.

Carrasco HJ, Frame IA, Valente SA, Miles MA 1996. Genetic exchange as a possible source of genomic diversity in sylvatic populations of Trypanosoma cruzi. Am J Trop Med Hyg 54: 418-424.

Clark CG 1992. Riboprinting: a molecular approach to the taxonomy of protozoa. In JJ Lee, AT Soldo (eds), Protocols in Protozoology, Allen Press, Lawrence, Kansas, p. D-4.1-D-4.4.

Clark CG, Diamond LS 1991. The Laredo strain and other Entamoeba histolytica-like amoebae are Entamoeba moshkovskii. Mol Biochem Parasitol 46: 11-18.

Clark CG, Pung OJ 1994. Host specificity of ribosomal DNA variation in sylvatic Trypanosoma cruzi from North America. Mol Biochem Parasitol 66: 175179.

Clark CG, Martin DS, Diamond LS 1995. Phylogenetic relationships among anuran trpanosomes as revealed by riboprinting. J Euk Microbiol 42: 92-96.

Cotton RGH 1997. Mutation Detection, Oxford University Press, Oxford, 198 pp.

Gasser RB, Zhu XQ 1999. Sequence-based analysis of enzymatically amplified DNA fragments by mutation detection techniques. Parasitol Today 15: 462465.

Hoare CA 1972. The Trypanosomes of Mammals - A Zoological Monograph, Blackwell Scientific Publications, Oxford, 749 pp.

Lerman LS, Silverstein T 1987. Computational simulation of DNA melting and its application to denaturing gradient gel electrophoresis. Methods Enzymol 155: 482-501.

Li W-H 1997. Molecular Evolution, Sinauer Associates, Inc., Massachusetts, 487 pp.

Macedo AM, Pena SDJ 1998. Genetic variability of Trypanosoma cruzi: implications for the pathogenesis of Chagas disease. Parasitol Today 14: 119-124.

Miles MA, Povoa MM, Souza AA, Lainson R, Shaw JJ, Ketteridge DA 1981. Chagas disease in the Amazon Basin: II. The distribution of Trypanosoma cruzi zymodemes 1 and 3 in Pará State, north Brazil. Trans $R$ Soc Trop Med Hyg 75: 667-674. 
Miles MA, Toyé PJ, Oswald SC, Godfrey DG 1977. The identification by isoenzyme patterns of two distinct strain-groups of Trypanosoma cruzi circulating independently in a rural area of Brazil. Trans $R$ Soc Trop Med Hyg 71: 217-225.

Momen H 1999. Taxonomy of Trypanosoma cruzi: a commentary on characterization and nomenclature. Mem Inst Oswaldo Cruz 94: 181-184.

Noyes HA 1998. Can trypanosome trees be trusted? Parasitol Today 14: 49-50.

Pulido M, Martinez-Calvillo S, Hernandez R 1996. Trypanosoma cruzi rRNA genes: a repeated element from the non-transcribed spacer is locus specific. Acta Trop 62: 163-170.

Schlötterer C 1995. Temperature-gradient gel electrophoresis as a screening tool for polymorphisms in multigene families. Electrophoresis 16: 722-728.

Sheffield VC, Beck JS, Nichols B, Cousineau A, Lidral AC, Stone EM 1992. Detection of multiallele polymorphisms within gene sequences by GC-clamped denaturing gradient gel electrophoresis. Am J Hum Gen 50: 567-575.

Souto RP, Vargas N, Zingales B 1999. Trypanosoma rangeli: discrimination from Trypanosoma cruzi based on a variable domain from the large subunit ribosomal RNA gene. Exp Parasitol 91: 306-314.

Stevens JR, Gibson WC 1999. The molecular evolution of trypanosomes. Parasitol Today 15: 432-437.

Stothard JR 2000. Trypanosome trees and homologies. Parasitol Today 16: 173.
Stothard JR, Frame IA, Miles MA 1997. Use of polymerase chain reaction-based single strand conformational polymorphism and denaturing gradient gel electrophoresis methods for detection of sequence variation of ribosomal DNA of Trypanosoma cruzi. Int J Parasitol 27: 339-343.

Stothard JR, Frame IA, Miles MA 1999. Genetic diversity and genetic exchange in Trypanosoma cruzi: dual drug-resistant "progeny" from episomal transformants. Mem Inst Oswaldo Cruz 94: 189-193.

Stothard JR, Frame IA, Carrasco HJ, Miles MA 1998a. On the molecular taxonomy of Trypanosoma cruzi using riboprinting. Parasitology 117: 243-247.

Stothard JR, Frame IA, Carrasco HJ, Miles MA 1998b. Temperature gradient gel electrophoresis (TGGE) analysis of riboprints from Trypanosoma cruzi. Parasitology 117: 249-253.

Tibayrenc M 1998a. Genetic epidemiology of parasitic protozoa and other infectious agents: the need for an integrated approach. Int J Parasitol 28: 85-104.

Tibayenc M 1998b. Integrated genetic epidemiology of infectious diseases: the Chagas model. Mem Inst Oswaldo Cruz, 93: 577-580.

Wright ADG, Li S, Feng S, Martin DS, Lynn DH 1999. Phylogenetic position of the kinetoplastids, Cryptobia bullocki, Cryptobia catostomi, and Cryptobia salmositica and monophyly of the genus Trypanosoma inferred from small subunit ribosomal RNA sequences. Mol Biochem Parasitol 99: 69-76. 